\title{
(2) OPEN ACCESS \\ Role of CT-guided transthoracic biopsy in the diagnosis of mycobacterial infection
}

\author{
Kyongmin Sarah Beck, ${ }^{\oplus 1}$ Dae Hee Han, ${ }^{1}$ Kyo Young Lee, ${ }^{2}$ Seung Joon $\mathrm{Kim}^{3}$
}

\begin{abstract}
'Department of Radiology, Seoul St Mary's Hospital, College of Medicine, The Catholic University of Korea, Seoul, Republic of Korea ${ }^{2}$ Department of Hospital Pathology, Seoul St Mary's Hospital, College of Medicine, The Catholic University of Korea, Seoul, Republic of Korea

${ }^{3}$ Division of Pulmonology, Department of Medicine, Seoul St Mary's Hospital, College of Medicine, The Catholic University of Korea, Seoul, Republic of Korea
\end{abstract}

\section{Correspondence to} Dr Kyongmin Sarah Beck, Department of Radiology, Seoul St. Mary's Hospital, College of Medicine, The Catholic University of Korea, Seocho-gu, Seoul, 06591, Republic of Korea; sallahbar@gmail.com

Accepted 7 December 2018 Published Online First 11 January 2019

\begin{abstract}
Mycobacterial infection(MI) is sometimes diagnosed using CT-guided transthoracic needle biopsy (TNB). However, the exact role of CT-guided TNB in this diagnostic process is not clearly known. The purpose of this study is to analyze the role of CT-guided TNB in patients with MI who present with a focal lung lesion. Of 1233 patients who underwent CT-guided TNB from January 2010 through February 2016 at our institution, patients with a final diagnosis of $\mathrm{MI}$ were included for analysis. Clinical characteristics and biopsy-related factors were compared between patients whose diagnosis could be established using TNB samples alone (group 1) and those whose samples from additional tests were necessary for diagnosis (group 2). We also analyzed the possible benefit of CT-guided TNB as compared with bronchoscopy in a subgroup who underwent both procedures. 47 patients with Ml were included in the study, with 37 patients $(78.7 \%)$ in group 1 and 10 patients $(21.2 \%)$ in group 2. There was no statistically significant difference in clinical characteristics or biopsy-related factors between the two groups. Of 41 patients with MI who underwent both bronchoscopy and TNB, success in diagnosis was solely attributable to TNB in 16 (39.0\%) patients; in $19(46.0 \%)$ patients, diagnosis could be made based on bronchoscopy results alone. MI can be successfully diagnosed by CT-guided TNB in about $80 \%$ of patients with MI who underwent TNB, but $46 \%$ of the patients could have been diagnosed with bronchoscopy results alone. CT-guided TNB is inferior to bronchoscopy in the differentiation of Mycobacterium species even in peripheral lung lesions.
\end{abstract}

\section{INTRODUCTION}

The diagnosis of tuberculosis (TB) or non-tuberculous mycobacterial (NTM) infection is conventionally made with sputum studies and bronchoscopy in most clinically suspected cases with compatible imaging findings. ${ }^{1}$ However, in some cases, TB or NTM infection can appear as a solitary pulmonary nodule (SPN) and is diagnosed by CT-guided transthoracic needle biopsy (TNB) of the lung. Additionally, in cases in which focal lung lesions coexist with imaging findings that may suggest mycobacterial infection and negative sputum study results, CT-guided TNB is sometimes considered as a diagnostic procedure.

\section{Significance of this study}

What is already known about this subject?

- Most mycobacterial infections are diagnosed on sputum study and bronchoscopy.

- Mycobacterial infection is sometimes diagnosed with CT-guided transthoracic needle biopsy (TNB).

- Mycobacterium tuberculosis (TB)infection and nontuberculous mycobacterial (NTM) infection cannot be differentiated morphologically on histopathologic specimen.

What are the new findings?

- Mycobacterial infection can be successfully diagnosed by CT-guided TNB in about $80 \%$ of patients who underwent TNB and were ultimately diagnosed with mycobacterial infection.

- About $46 \%$ of the patients who undergo CT-guided TNB and were diagnosed with mycobacterial infection were also diagnosed on bronchoscopy, which means those patients could have avoided an invasive procedure such as CT-guided TNB.

- Specimen TB-PCR is very helpful both in making a diagnosis and in differentiating TB and NTM infection.

- Even peripheral focal lung lesions can be successfully diagnosed with bronchoscopy.

How might these results change the focus of research or clinical practice?

- Bronchoscopy should be first performed before considering a CT-guided TNB for focal lung lesions suspected of mycobacterial infection, even in peripheral lung lesions.

- Specimen TB-PCR should be done when performing a CT-guided TNB with a high clinical suspicion of benign, infectious lesion.

There are a few studies on CT-guided TNB and diagnosis of $\mathrm{TB}^{2}{ }^{3}$ but none has specifically addressed the role of CT-guided TNB in the diagnosis of TB. Also, many of the previous studies involving histologic specimens for the diagnosis of $\mathrm{TB}^{3}{ }^{4}$ overlooked the fact that TB and NTM cannot be differentiated 
morphologically on histopathology, ${ }^{56}$ and included patients solely based on histopathology, which we believe to be a limitation of those studies. Even if tissue acquired by CT-guided TNB shows histopathologic characteristics of mycobacterial infection, such as epithelioid cells, multinucleated giant cells or caseous necrosis, there are many additional steps to the final diagnosis of TB or NTM infection. However, the percentage of those patients finally diagnosed to be TB or NTM infection, after exactly what other procedures or how much time, and the role of CT-guided TNB in this diagnostic process are not clearly known. Additionally, there are cases in which CT-guided TNB fails to provide histopathologic diagnosis of mycobacterial infection, but TB or NTM infection is ultimately proven by other means, such as bronchoscopy and surgery. Without considering those cases, any assessment of the role of CT-guided biopsy in the diagnosis of mycobacterial infection would be incomplete. Moreover, because majority of the diagnoses of TB or NTM infection are aided by bronchoscopy, investigating the possible benefit of CT-guided TNB as compared with bronchoscopy seems necessary.

The purpose of this study is to investigate the role and possible benefit of CT-guided TNB in the diagnosis of TB and NTM infection.

\section{MATERIALS AND METHODS}

\section{Study population and data collection}

We retrospectively reviewed percutaneous CT-guided TNB-both core needle biopsy and aspiration-performed from January 2010 through February 2016 at our institution. From April 2010 to February 2016, 1233 patients underwent percutaneous CT-guided TNB. The histopathologic reports of all 1233 patients were searched for the phrase 'Mycobacterial infection' or phrases indicating a non-specific benign result, because at our hospital the pathologists only use the term 'Mycobacterial infection' instead of 'TB' or 'NTM infection' on histopathologic reports, because TB and NTM infection cannot be differentiated morphologically on histopathology. ${ }^{56}$ The patients who met either criterion were selected for detailed review of medical records. For each of these patients, we determined whether or not the patient was definitively diagnosed with either TB or NTM infection, based on the results of culture or PCR test from one or more samples obtained by means of TNB, bronchoscopy, sputum collection, or surgery.

Demographic and clinical features of the study population were collected from the electronic medical records. Patients' age, sex, history of TB, and follow-up and management after biopsy (including whether they had received anti-TB medication or not, the duration of anti-TB medication, and so on) were obtained. Several biopsy-related features were also obtained from the operator's report in our radiology database. Lesion size (longest diameter), lesion location (lobe), number of core biopsy specimens, pleura-to-target distance, and biopsy-related complications, such as pneumothorax, at postprocedural CT scan (present or absent) were documented. We also reviewed the subsequent imaging studies for the interval changes in the biopsied lesions. In addition, we searched the medical records for the number of positive results for mycobacterium tuberculosis (MTB) or NTM on culture or
PCR from specimens obtained from sputum collection and bronchoscopy from January 2010 through February 2016 to compare the number of patients with TNB-diagnosed mycobacterial infection with that of the entire mycobacterial infection population.

\section{CT-guided needle biopsy procedure}

All CT-guided TNBs were performed using a multidetector CT scanner (Siemens Definition AS Plus, Siemens Healthcare, Erlangen, Germany) with no CT-fluoroscopy function. All procedures were performed using the coaxial technique by one of four chest radiologists. Prior to biopsy, a preprocedural CT scan was initially performed to determine the most optimal and safest needle path to the target. Thereafter, an 18-gauge coaxial introducer was inserted under intermittent CT guidance. After confirming the proper placement of the needle tip within the target, tissue biopsy was conducted with a cutting needle (Stericut, TSK Laboratory, Tochigi, Japan). Occasionally, additional samples for culture were aspirated using the coaxial introducer after core needle biopsy was performed, at the radiologist's discretion. After the removal of the coaxial introducer, postprocedural CT was performed to identify immediate procedure-related complications, such as pneumothorax or perilesional hemorrhage. An erect chest radiograph was routinely obtained 3 hours after biopsy to evaluate the presence of pneumothorax.

\section{Bronchoscopy procedure}

Bronchoscopic examinations were performed without fluoroscopic guidance by one of five full-time faculty staffs with clinical experience in bronchoscopy for at least 1 year in the pulmonology division. Bronchoscopy (Model BF-1T260; Olympus, Tokyo, Japan) was performed via the transnasal or transoral approach. Midazolam was administered to achieve moderate sedation. Four per cent lidocaine was injected during the inspection of the vocal cord to anesthetize the upper airway as well as the tracheobronchial tree. All segments of the bronchus were observed. Next, the bronchoscope was moved to the bronchial segment of a target lesion located on CT to collect bronchial washing sample for TB-PCR, acid fast bacilli staining, and culture. TB-PCR was done using Xpert MTB/RIF assay (Cepheid, Sunnyvale, California, USA) for a rapid detection of TB.

\section{Bronchoscopy versus CT-guided TNB}

The pulmonologists at our institution routinely do bronchoscopy on the same day as CT-guided TNB for the evaluation of SPN regardless of suspected benignity or malignancy, which made evaluating the added benefit of CT-guided TNB as compared with bronchoscopy possible. We recorded whether the patients had undergone bronchoscopy in addition to CT-guided TNB, whether TB-PCR or culture was done with samples taken from bronchoscopy and CT-guided TNB, the results, and the time taken for the culture and PCR results to come out. We also recorded the time for the histopathologic diagnosis of TNB specimen to be made. We then compared the positive rates of each study and the median time taken for the diagnosis. 


\section{Histopathology}

Histopathologic diagnosis of mycobacterial infection was made when the tissue showed chronic granulomatous inflammation, with or without caseous necrosis. When chronic granulomatous inflammation was observed, ZiehlNeelsen staining was additionally done. TB-PCR was performed on the extracted DNA from formalin-fixed, paraffin-embedded tissue using a MTB PCR kit (Biocore, Seoul, Korea), which targets a part of the IS6110 gene for amplification, according to the manufacturer's instructions. When TB-PCR test was positive, the result was separately reported alongside the histopathology report. NTM PCR on tissue samples is currently not available at our hospital, so only TB-PCR was done using the tissue samples when requested by the clinician or deemed appropriate by the pathologist.

\section{Statistical analysis}

We compared the clinical characteristics and biopsy-related factors between patients in which the diagnosis could be established using TNB samples alone (group 1) and those in which samples from additional tests were necessary for the diagnosis (group 2). Pearson's $X^{2}$ or Fisher's exact test was used for categorical variables, and the independent t-test or Mann-Whitney U test was used for continuous variables, as appropriate. Statistical analyses were performed at a significance level of 0.05 using SPSS V.24.0.

\section{RESULTS}

\section{Patient characteristics}

Forty-seven patients who underwent CT-guided TNB from January 2010 through February 2016 were ultimately diagnosed with mycobacterial infection, which includes MTB, NTM infection, and unclassified mycobacterial infection (UMI). There were 25 male and 22 female patients, with a mean age of 53 years. During the same period, 3919 patients were diagnosed with MTB or NTM infection on sputum studies and bronchoscopy.

\section{TNB diagnosis of mycobacterial infection and biopsy- related characteristics}

Of 47 patients ultimately diagnosed with mycobacterial infection, there were 37 patients $(78.7 \%)$ in group 1 and 10 patients $(21.2 \%)$ in group 2 . In group 1,28 patients $(75.7 \%)$ were diagnosed histopathologically and 9 patients $(24.3 \%)$ originally had non-specific benign results on histology but tested positive on TB-PCR $(n=6)$ or culture $(n=3)$ using TNB specimen. In group $2(n=10)$, the diagnosis was established either on surgery $(n=5)$, sputum culture $(n=2)$, or bronchoscopy $(n=3 ; n=3$ and $n=2$ for bronchial washing fluid culture and TB-PCR, respectively). Overall, 19 out of 47 patients $(40.4 \%)$ originally had non-specific benign results on histology but were ultimately diagnosed to be mycobacterial infection.

The median size of the biopsied lesion was $3.2 \mathrm{~cm}$, with a median pleura-to-target distance of $1.1 \mathrm{~cm}$. The median number of cores obtained was 4 . Nine out of 47 patients (19.1\%) had developed pneumothorax after CT-guided TNB, but all of them were asymptomatic and none required intervention (such as chest tube placement or pain control).
Table 1 Results of univariate analyses between group 1 and group 2

\begin{tabular}{|c|c|c|c|}
\hline Variable & Group $1(n=37)$ & Group $2(n=10)$ & $P$ value \\
\hline Age $^{*}$ (years) & $53.1 \pm 15.8$ & $54.3 \pm 18.0$ & 0.544 \\
\hline Sex & & & 0.820 \\
\hline Male & 20 & 5 & \\
\hline Female & 17 & 5 & \\
\hline Diagnosis & & & 0.456 \\
\hline MTB or NTM & 27 & 6 & \\
\hline $\begin{array}{l}\text { Unspecified mycobacterial } \\
\text { infection }\end{array}$ & 10 & 4 & \\
\hline History of TB & & & $>0.999$ \\
\hline Yes & 8 & 2 & \\
\hline No & 29 & 8 & \\
\hline Anti-TB medication & & & $>0.999$ \\
\hline Yes & 29 & 8 & \\
\hline No & 8 & 2 & \\
\hline Emphysema & & & 0.285 \\
\hline Yes & 5 & 2 & \\
\hline No & 34 & 8 & \\
\hline $\mathrm{CT}$ findings & & & 0.456 \\
\hline Solitary nodule or mass & 10 & 4 & \\
\hline $\begin{array}{l}\text { Nodule or mass with } \\
\text { satellite nodules }\end{array}$ & 27 & 6 & \\
\hline Lesion sizet $(\mathrm{cm})$ & $3.1(2.3-4.0)$ & $2.7(2.1-3.6)$ & 0.547 \\
\hline $\begin{array}{l}\text { Pleura-to-target distancet } \\
(\mathrm{cm})\end{array}$ & $1.2(0.0-2.1)$ & $1.0(0.0-2.2)$ & 0.990 \\
\hline Number of cores $t$ & $4.0(3.0-4.5)$ & $4.0(2.8-4.0)$ & 0.600 \\
\hline Biopsy location & & & $>0.999$ \\
\hline Upper lobes & 20 & 6 & \\
\hline Middle and lower lobes & 17 & 4 & \\
\hline TB-PCR on TNB specimen & & & 0.277 \\
\hline Done & 16 & 2 & \\
\hline Not done & 21 & 8 & \\
\hline Pneumothorax & & & 0.377 \\
\hline Yes & 6 & 3 & \\
\hline No & 31 & 7 & \\
\hline
\end{tabular}

* Data are mean \pm SD

tData are median (with IQR in parentheses).

MTB, Mycobacterium tuberculosis; NTM, non-tuberculous mycobacterial infection; TB, tuberculosis; TNB, transthoracic needle biopsy.

Table 1 summarizes the clinical characteristics and biopsy-related factors of the study population with the results of univariate analyses between group 1 and group 2 . None of the clinical characteristics and biopsy-related factors was significantly different between group 1 and group 2 .

\section{Bronchoscopy versus TNB in the diagnosis and differentiation of TB and NTM infection}

There were 42 patients who underwent both TNB and bronchoscopy. There was one patient who underwent bronchoscopy but did not have bronchial washing samples collected, which is not a norm at our hospital so we excluded the patient from further analysis.

Out of 41 patients, $35(85.3 \%)$ showed positive results for mycobacterial infection either on TNB or bronchoscopy. Overall, mycobacterial infection was diagnosed by TNB in 32 patients $(78.0 \%)$ and by bronchoscopy in 19 patients $(46.0 \%)$; in 16 patients, TNB was the sole positive test $(39.0 \%)$ for mycobacterial infection; in 16 patients (39.0\%), both bronchoscopy and TNB were positive; in the 
Table 2 Various study results according to various sampling methods

\begin{tabular}{|c|c|c|c|c|}
\hline & TNB & Bronchoscopy & Surgery* & Sputum study* \\
\hline \multicolumn{5}{|l|}{ Histopathology (H\&E slides) } \\
\hline Mycobacterial infection & 28 & NA & 6 & NA \\
\hline Non-specific benign & 19 & NA & 0 & NA \\
\hline \multicolumn{5}{|l|}{ TB-PCR } \\
\hline Positive & 10 & 10 & 2 & 0 \\
\hline Negative & 8 & 31 & 3 & 2 \\
\hline Not done & 29 & 1 & 1 & 17 \\
\hline \multicolumn{5}{|l|}{ Culture } \\
\hline Positive & 5 (3 TB, 2 NTM infection) & 18 (13 TB, 5 NTM infection) & $1(\mathrm{~TB})$ & 3 (1 TB, 2 NTM infection) \\
\hline Negative & 17 & 23 & 2 & 3 \\
\hline Not done & 25 & 1 & 3 & 13 \\
\hline Total & 47 & 42 & 6 & 19 \\
\hline
\end{tabular}

*Excluding patients with positive TB-PCR or culture results on TNB or bronchoscopy.

NA, not available; NTM, non-tuberculous mycobacterial infection; TB, tuberculosis; TNB, transthoracic needle biopsy.

remaining 3 patients $(7.3 \%)$, bronchoscopy was the sole positive test. The remaining seven patients had their final diagnoses confirmed either on surgery $(n=5)$ or sputum culture $(n=2)$.

Out of 41 patients 10 (24.4\%) and 18 (43.9\%) showed positive results on TB-PCR and culture using bronchial washing samples, respectively. Nineteen patients showed positive results on either TB-PCR or culture using bronchial washing samples. TB-PCR was done using TNB specimen in 18 patients, and 10 of them (55.6\%) showed positive results. Culture was done using TNB specimen (mostly aspirates) in 22 out of 47 patients, and 5 of them (29.4\%) showed positive results. Those who were positive on TB-PCR using TNB specimen either did not show positive results on culture $(n=2)$ or did not have culture study done $(n=8)$, and the five patients with positive culture results did not have TB-PCR done using TNB specimen. Table 2 summarizes the results of various studies according to various sampling methods.

Differentiation of TB and NTM infection was possible in 32 out of 41 patients $(78.0 \%)$, by means of bronchoscopy in 19 (46.3\%; 10 TB-PCR-positive cases and 18 bronchial washing culture-positive cases), TNB in 15 (36.6\%; 10 TB-PCR-positive cases and 5 culture-positive cases), and both bronchoscopy and TNB in 6. The other four were identified on sputum study $(n=2)$ and surgery $(n=2)$. Ultimately, 24 patients (58.5\%) were finally classified as MTB infection and 8 patients (19.5\%) as NTM infection. The other 10 patients $(24.4 \%)$ remained as UMI.

Bronchoscopy was mostly done on the same day as TNB (median difference of 0 day), and the results of TB-PCR using bronchial washing samples were mostly available on the day of bronchoscopy, hence on the same day as TNB. The histopathologic results of TNB and TB-PCR using TNB samples were available at a median of 3 days and 8 days after biopsy, respectively. Positive results of culture using bronchial washing and TNB samples were available at a median of 16 days after bronchoscopy and 14 days after TNB, respectively. Time to earliest identification of TB or NTM infection using bronchoscopy and TNB (positive results on PCR or culture, whichever came out first) was at a median of 1 day and 9 days, respectively.

\section{Management and follow-up}

Of these 47 patients, 37 received anti-TB medication, which includes 22 patients with TB, 11 UMI, and 4 patients who were later diagnosed with NTM infection; 8 did not receive anti-TB medication (1 TB, 2 UMI, and 4 NTM infection); and 2 were lost to follow-up. Of 37 patients who received anti-TB medication, 26 (18 TB, 6 UMI, and 2 NTM infection) showed regression of the lesion on follow-up CT; 3 patients (1 TB, 1 UMI, and 1 NTM infection) were stable on follow-up CT and 8 patients (4 UMI, 3 TB, and 1 NTM infection) underwent surgical removal of the lesion. Of eight patients who did not receive anti-TB medication, five showed regression of the lesion on follow-up CT (1 UMI and 4 NTM infection); 1 patient with UMI was stable on follow-up CT; and 2 patients (1 TB and 1 NTM infection) showed aggravation of the lesion. The patient with TB who showed aggravation of the lesion did not receive anti-TB medication because he had end-stage bladder cancer and expired 9 months after the biopsy.

\section{DISCUSSION}

In this study, we have revealed that CT-guided TNB (H\&E staining, PCR, culture) was diagnostic for mycobacterial infection in 37 patients over the course of 6 years, which is a much smaller number compared with that of patients with mycobacterial infection diagnosed by means of sputum studies or bronchoscopy during the same period (ratio 1:105.9). These 37 patients account for $78.7 \%$ of the 47 patients with confirmed mycobacterial infection who underwent TNB. This may seem effective, but with a more careful analysis incorporating the results of bronchoscopy a slightly different conclusion can be made. In 41 patients who underwent both bronchoscopy and CT-guided TNB, about $40 \%$ of the patients showed positive results on both bronchoscopy and CT-guided TNB, and the overall diagnostic yield of bronchoscopy was $46.0 \%$ in that population. In other words, $46.0 \%$ of the 41 patients could have reached a diagnosis by undergoing bronchoscopy alone, without the help of CT-guided TNB. CT-guided TNB is a much more invasive procedure than bronchoscopy; the overall pooled complication rate of CT-guided TNB is about 38\%, with 
$25 \%$ risk of pneumothorax, $18 \%$ risk of pulmonary hemorrhage, and $6 \%$ risk of major complication, which includes air embolism and death, ${ }^{7}$ whereas the rate of pulmonary complications of bronchoscopy is much lower, with less than $0.2 \%$ of pneumothorax and $0.2 \% \sim 0.3 \%$ of respiratory failure. ${ }^{8}$ Thus we believe it is reasonable to avoid CT-guided-TNB if possible and reserve it for circumstances in which diagnosis cannot be established with less invasive methods.

Another problem of making a diagnosis of mycobacterial infection with CT-guided-TNB is that majority $(75.7 \%)$ of the diagnoses of mycobacterial infection made using TNB specimens in our study were through histopathologic examination, and only 10 and 5 patients had positive results on specimen TB-PCR and culture results, respectively. Without the TB-PCR or culture results, differentiation of TB and NTM cannot be made histopathologically on the H\&E slides. ${ }^{56}$ In contrast, bronchoscopic results are either from TB-PCR or culture study, which can differentiate TB and NTM. Because the management of TB and NTM greatly differs, ${ }^{9-11}$ one cannot start treatment based solely on the histopathologic evidence of 'Mycobacterial infection', which may turn out to be either TB or NTM. Therefore, even with positive TNB results that come out at a median of 3 days after the biopsy, the clinicians still have to refer to bronchoscopy results for further information regarding the management of the patient. Moreover, about 50\% of the positive bronchoscopy results were from TB-PCR study using Xpert MTB/RIF assay, which takes a median of 1 day for the results to come out, and that is not a long time to wait for. Bronchial washing culture results take a median of 2 weeks, but with a promise of differentiation of TB and NTM. In fact, there are four cases of NTM infection that were mistaken as TB based on histopathology results alone, leading to unnecessary anti-TB medication. Of these four patients with NTM, two patients even showed regression of the lesions on follow-up CTs, indicating that differentiation of TB and NTM cannot be made by apparent response to anti-TB medication and histopathology results alone. Altogether, these cases emphasize the importance of differentiation of TB and NTM and highlight the limitation of histopathology results of TNB specimen, which could mislead the diagnosis and treatment altogether.

Moreover, due to the invasive nature of CT-guided TNB, procuring additional specimens for culture is often difficult and done with caution during TNB, as evidenced by the fact that only 22 of 47 patients $(46.8 \%)$ had additional TNB samples for culture. Compared with that, bronchial washing can easily provide ample samples for further tests; all but one patient who underwent bronchoscopy had bronchial washing specimens ready for both culture and TB-PCR. In addition to differentiating TB and NTM, an ample amount of samples are needed for further identification of NTM species using additional PCR tests, because it is important to know the exact NTM species in order to determine the management of the disease. ${ }^{10}{ }^{11}$ NTM PCR using tissue specimen is not widely used, ${ }^{12}$ also limiting the role of CT-guided TNB in the differentiation and identification of mycobacterial species.

Despite undergoing both bronchoscopy CT-guided TNB, 10 patients ( 3 of them additionally received surgery) remained as UMI. It is still unclear how these cases should be managed. Although a therapeutic trial of anti-TB medication was done for 9 of these 10 patients, we do not know whether it was necessary or not. Five of them did show regression on follow-up CT, but it is not certain whether anti-TB medication played a role in that change, because one patient remained stable despite anti-TB medication and another UMI case showed spontaneous regression of the lesions without anti-TB treatment. Future study with a larger number of patients is necessary to determine the management of patients with UMI.

One thing of note is that TB or NTM infection was the first considered diagnosis or in the differential diagnosis on radiologic reports made by thoracic radiologists at our hospital for pre-TNB chest CTs of 31 and 7 out of 47 patients, respectively. Many of these patients showed satellite nodules around the 'SPN' on chest CT images. The major reason (25 out of 38 patients) for proceeding with CT-guided TNB in these patients despite the radiologic reports was the clinicians' wish to rule out malignancythey wanted the histopathologic proof of benignity. In that aspect, CT-guided TNB is an excellent tool because it could provide a specific benign diagnosis to almost $80 \%$ of the patients who underwent the procedure.

As for the technical aspects of TNB in the study population, the typical target was a peripherally located focal lung lesion (median pleura-to-target distance of $1.1 \mathrm{~cm}$ ). Many clinicians request CT-guided TNB because they believe bronchoscopy would not be helpful in the diagnosis of a peripheral lesion, for conventional fiberoptic bronchoscopy can only access the central airways, and the sensitivity for peripheral lung nodule using traditional transbronchial sampling techniques is less than $20 \%{ }^{13}$ However, our study results show that even in peripheral lung lesions close to the pleura, the diagnostic yield of bronchoscopy was almost $50 \%$. This is probably due to the fact that we have evaluated the yield of bronchial washing samples rather than direct bronchial aspiration biopsy of the peripherally located target lesion. Our results show that TB-PCR on TNB specimens played a very important role in the diagnosis of TB. Although there was no significant difference, a higher percentage of patients in group 1 underwent TB-PCR on the TNB specimen compared with those in group 2. Additionally, in about $30 \%$ of patients who had non-specific benign results on histologic diagnosis (H\&E), the final diagnosis was established because of the positive result on TB-PCR on the TNB specimen. It took a median of 6 days for the TNB specimen to produce TB-PCR results because ordering of the test was not done until the pathologist recommended it. Thus, we believe that in an endemic area, TB-PCR should be considered in all TNB specimens with benign results. This would also help further differentiate TB and NTM infection on TNB specimens, for mycobacterial infection with negative TB-PCR results could be presumed to be NTM infection.

This study was possible because at our institution bronchoscopy and TNB are often done concurrently. However, for physicians preferring a serial approach, we would recommend that bronchoscopy be first considered even in a peripheral lesion if mycobacterial infection is suspected as the cause, and that TNB should be reserved for cases with a non-diagnostic bronchoscopy. The management of a patient does not end with establishing a specific benign 
diagnosis, and the patient still has to receive medication accordingly based on further test results. In that aspect, bronchoscopy is a safe and less invasive procedure that can be easily done and provide results that are both diagnostic and have an impact on the management of the patient. Our study dealt with patients with a focal lung lesion, which is not an image finding for active TB but rather signifies a granuloma. ${ }^{1}$ A granuloma is a host-defense mechanism that contains and shelters the bacteria before spreading and reactivation, ${ }^{14}$ suggesting low infectivity, and waiting for 2 weeks would not cause significant delay or harm to the management of the patient.

Our study has several limitations. First, it is a retrospective study done at one institution with a very small sample size. Practice pattern could vary among different clinicians, pathologists, and intervention radiologists in other institutions. However, we hope this could provide useful information even for those with a different practice pattern. Second, the study may have selection bias because the patients who undergo CT-guided TNB only represent a very small subgroup of patients with MTB and NTM infection. Third, there are only five positive cases of TNB-specimen culture study, and this is because core biopsy technique is preferred by the chest radiologists at our institution and aspiration technique is only seldom used.

In conclusion, mycobacterial infection can be successfully diagnosed by CT-guided TNB in about $80 \%$ of patients who underwent TNB and were ultimately diagnosed with mycobacterial infection, but $46 \%$ of the patients could have been diagnosed with bronchoscopy results alone. CT-guided TNB is inferior to bronchoscopy in the differentiation of Mycobacterium species even in peripheral lung lesions. Therefore we recommend waiting for the bronchoscopic study results before considering CT-guided TNB when there is a possibility of mycobacterial infection as the cause of a focal lung lesion.

Contributors Conception and design: KSB, DHH. Administrative support: $\mathrm{DHH}$. Provision of study materials or patients: all authors. Collection and assembly of data: $\mathrm{KSB}, \mathrm{DHH}$. Data analysis and interpretation: $\mathrm{KSB}, \mathrm{DHH}$. Manuscript writing: all authors. Final approval of the manuscript: all authors.

Funding The authors have not declared a specific grant for this research from any funding agency in the public, commercial or not-for-profit sectors.

Competing interests None declared.

Patient consent Not required.
Ethics approval This retrospective analysis was approved by the Institutional Review Board of the Seoul St Mary's Hospital, and the requirement for written informed consent was waived.

Provenance and peer review Not commissioned; externally peer reviewed. Data sharing statement There are no additional unpublished data from the study.

Open access This is an open access article distributed in accordance with the Creative Commons Attribution Non Commercial (CC BY-NC 4.0) license, which permits others to distribute, remix, adapt, build upon this work noncommercially, and license their derivative works on different terms, provided the original work is properly cited, an indication of whether changes were made, and the use is non-commercial. See: http://creativecommons.org/ licenses/by-nc/4.0/.

\section{REFERENCES}

1 Jeong YJ, Lee KS, Yim J-J. The diagnosis of pulmonary tuberculosis: a Korean perspective. Precision and Future Medicine 2017;1:77-87.

2 Choo JY, Lee KY, Kim MY, et al. Pulmonary tuberculosis confirmed by percutaneous transthoracic needle biopsy: analysis of CT findings and review of correlations with underlying lung disease. Balkan Med J 2014;31:208-13.

3 Fukuda H, Ibukuro K, Tsukiyama T, et al. CT-guided transthoracic core biopsy for pulmonary tuberculosis: diagnostic value of the histopathological findings in the specimen. Cardiovasc Intervent Radiol 2004;27:226-30.

$4 \mathrm{Cha} \mathrm{JH}, \mathrm{Han}$ J, Park HJ, et al. Aneurysmal appearance of medium-sized bronchi: a peripheral manifestation of endobronchial tuberculosis. AJR Am J Roentgenol 2009;193:W95-9.

5 Mukhopadhyay S, Gal AA. Granulomatous lung disease: an approach to the differential diagnosis. Arch Pathol Lab Med 2010;134:667-90.

6 Mukhopadhyay S. Role of histology in the diagnosis of infectious causes of granulomatous lung disease. Curr Opin Pulm Med 2011;17:189-96.

7 Heerink WJ, de Bock GH, de Jonge GJ, et al. Complication rates of CT-guided transthoracic lung biopsy: meta-analysis. Eur Radiol 2017;27:138-48.

8 Stahl DL, Richard KM, Papadimos TJ. Complications of bronchoscopy: a concise synopsis. Int J Crit IIIn Inj Sci 2015;5:189-95.

9 Nahid P, Dorman SE, Alipanah N, et al. Official american thoracic society/ centers for disease control and prevention/infectious diseases society of america clinical practice guidelines: treatment of drug-susceptible tuberculosis. Clin Infect Dis 2016;63:e147-95.

10 Griffith DE, Aksamit T, Brown-Elliott BA, et al. An official ATS/IDSA statement: diagnosis, treatment, and prevention of nontuberculous mycobacterial diseases. Am J Respir Crit Care Med 2007;175:367-416.

11 Ryu YJ, Koh WJ, Daley CL. diagnosis and treatment of nontuberculous mycobacterial lung disease: clinicians' perspectives. Tuberc Respir Dis 2016;79:74-84

12 Kim YN, Kim KM, Choi HN, et al. Clinical Usefulness of PCR for differential diagnosis of tuberculosis and nontuberculous mycobacterial infection in paraffin-embedded lung tissues. J Mol Diagn 2015;17:597-604.

13 Arenberg D. Electromagnetic navigation guided bronchoscopy. Cancer Imaging 2009;9:89-95.

14 Silva Miranda M, Breiman A, Allain S, et al. The tuberculous granuloma: an unsuccessful host defence mechanism providing a safety shelter for the bacteria? Clin Dev Immunol 2012;2012:1-14. 